\title{
SCS macrophages protect the CNS
}

\begin{abstract}
Little is known about how the immune system prevents vesicular stomatitis virus (VSV) from entering the central nervous system (CNS) following infection through the natural subcutaneous route. A study published in Nature now shows that subcapsular sinus (SCS) macrophages in the draining lymph nodes prevent CNS entry by VSV following subcutaneous infection through the production of type I interferons (IFNs), which act on both haematopoietic and stromal cells, including intranodal nerve cells.

SCS macrophages have an important role in viral clearance and initiation of antiviral humoral immunity, but it is not known whether they have a role in preventing virus entry into the CNS. SCS macrophages were selectively depleted from the draining (popliteal) lymph node of mice by injection of clodronate liposomes (CLLs) in the footpad. Infection of these mice in the footpad with a low dose of VSV 6 days later resulted in ascending CNS pathology and mortality in $\sim 60 \%$ of mice by day 10 post-infection. By contrast, almost all control mice survived VSV infection.

The virus was detected in the brain and spinal cord of CLLtreated but not control mice 7 days post-infection.

By using VSV that expresses enhanced green fluorescent protein during replication, the authors showed that SCS macrophages in the draining lymph node are the main site of viral replication following subcutaneous infection, and there was no evidence of viral replication in the adjacent nerves. By contrast, viral replication occurred predominantly in the intranodal nerves in the absence of SCS macrophages.

Further analysis showed that the adaptive immune response to VSV was not compromised in CLL-treated mice; so how do SCS macrophages protect against neuroinvasion? The lymph nodes of VSV-infected CLL-treated mice had significantly reduced levels of IFNa ( 90\%) compared with controls. The depletion of plasmacytoid dendritic cells (pDCs) in VSV-infected wild-type mice resulted in a $~ 50 \%$ reduction in IFNa levels and depletion of both pDCs and SCS macrophages completely abrogated IFNa production, indicating that both cell types are sources of IFNa in response to VSV infection. However, in contrast to macrophage depletion, antibody-mediated depletion of $\mathrm{pDCs}$ prior to VSV
\end{abstract}

infection did not result in increased mortality in either control or CLLtreated mice, suggesting that localized IFNa production by SCS macrophages alone is neuroprotective. IFNa from pDCs seems to be dispensable for survival after VSV infection but may contribute to other aspects of antiviral immunity, such as the development of adaptive immune responses.

Finally, the authors generated chimeric mice in which either haematopoietic or stromal cells lacked expression of the type I IFN receptor (IFNAR). Mice lacking haematopoietic cell IFNAR expression died abruptly 4-5 days after VSV infection, and viral replication occurred in numerous lymph node cells. VSV-infected mice lacking stromal cell IFNAR expression developed ascending CNS pathology in a similar way to CLL-treated wild-type mice, and virus replication was restricted to SCS macrophages. These data indicate that type I IFNs must act on both haematopoietic and stromal cells (including intranodal nerves) to prevent lethal VSV infection.

These results identify SCS macrophages as crucial gatekeepers to the CNS that prevent fatal viral neuroinvasion upon peripheral infection through the production of type I IFNs.

Olive Leavy

ORIGINAL RESEARCH PAPER lannacone, M et al. Subcapsular sinus macrophages prevent CNS invasion on peripheral infection with a neurotropic virus. Nature 465, 1079-1083 (2010) 